\title{
Reflexões sobre instrumentos básicos de avaliação psicológica em uso no Brasil
}

\author{
Reflections on basic psychological assessment instruments in use in Brazil \\ Reflexiones sobre los instrumentos básicos de evaluación psicológica em uso em Brasil
}

Recebido: 11/11/2021 | Revisado: 20/11/2021 | Aceito: 24/11/2021 | Publicado: 06/12/2021

Lilian Regina Simões

ORCID: https://orcid.org/0000-0002-7609-1813 Secretaria de Estado de Educação, Brasil E-mail: lilian.simoes@unemat.br

Mayara Rossi

ORCID: https://orcid.org/0000-0002-9182-1888 Instituto Federal de Mato Grosso, Brasil

E-mail: professoramayararossi@ hotmail.com

Estêveno de Freitas Rodrigues

ORCID: https://orcid.org/0000-0002-0031-3619 Instituto Federal de Mato Grosso, Brasil

E-mail: estevenorodrigues@hotmail.com

Vanusa Maria de Oliveira

ORCID: https://orcid.org/0000-0001-8901-3374 Instituto Federal de Mato Grosso, Brasil E-mail: vanusa.oliveira_@hotmail.com

Paula Daniella Leão Braun

ORCID: https://orcid.org/0000-0002-2442-3257 Secretaria de Educação do Estado do Mato Grosso, Brasil E-mail: paulabraun_nx@hotmail.com Aparecida Rodrigues da Silva

ORCID: https://orcid.org/0000-0001-6334-7894 Secretaria de Estado de Educação, Brasil E-mail: rodriguescida028@gmail.com

Nilvana Gomes da Silva

ORCID: https://orcid.org/0000-0002-6577-1717 Secretaria de Estado de Educação, Brasil E-mail: nilvana_gomes@hotmail.com

Pâmela dos Reis

ORCID: https://orcid.org/0000-0002-5580-540X Secretaria de Estado de Educação, Brasil E-mail: pam2019reis@gmail.com

Eva Lúcia Freitas

ORCID: https://orcid.org/0000-0002-4915-1681 Secretaria de Estado de Educação, Brasil E-mail: eva.lfreitas@hotmail.com Elaine Silva Santos

ORCID: https://orcid.org/0000-0002-3058-5412 Secretaria de Estado de Educação, Brasil

E-mail: elainesilvasantos15@hotmail.com

Edson Garcia da Silva

ORCID: https://orcid.org/0000-0002-2497-5449 Secretaria de Estado de Educação, Brasil E-mail: garciasilva140782tjp@gmail.com

Juliana Aparecida Reis Marcelino

ORCID: https://orcid.org/0000-0001-6795-9425 Secretaria de Estado de Educação, Brasil E-mail:marcelinoju2@gmail.com

Lamara Laís Marin Artuzi

ORCID: https://orcid.org/0000-0002-9743-4846 Secretaria de Estado de Educação, Brasil E-mail:lamaramarin1@gmail.com

\section{Resumo}

O objetivo principal deste estudo foi refletir sobre a aplicação dos testes psicológicos em uso no Brasil com as possibilidades de validação dos mesmos pela Sociedade Brasileira de Psicologia, evidenciando a sua utilidade e as principais incorrências em relação ao seu uso, principalmente no tocante as situações que evidenciam distúrbios 
relacionados as dificuldades de aprendizagem escolar em crianças com inteligência normal, por meio de uma avaliação compreensiva integrando o funcionamento intelectual e a organização da personalidade. A fundamentação teórica psicanalítica sustenta que processos psíquicos patológicos podem diminuir a função da curiosidade e causar sintomas de inibição intelectual ou falta de concentração. A utilização de instrumentos de avaliação psicológica é uma prática necessária para a atuação do psicólogo. Nessa perspectiva, esse estudo teve como objetivo investigar o uso de instrumentos de avaliação psicológica por psicólogos. Em relação aos instrumentos mais utilizados, constatou-se que são os testes de avaliação da personalidade.

Palavras-chave: Testes psicológicos; Formação profissional; Psicometria.

\begin{abstract}
The main objective of study was to reflect on the application of psychological tests in use in Brazil with the possibilities of validating them by the Brazilian Psychological Society, highlighting regarding the situations that show disorders related to school learning difficulties in children with normal intelligence, through a comprehensive assessment integrating intellectual functioning and personality organization. The psychoanalytical theoretical foundation holds that pathological psychic processes can decrease the function of curiosity and cause symptoms of intellectual inhibition or lack of concentration. The use of psychological assessment instruments is a necessary practice for the psychologist. In this perspective, this study aimed to investigate the use of psychologist. In this perspective, this study aimed to investigate the use of psychological assessment instruments by psychologists. Regarding the most used instruments, it was found that they are the personality assessment tests.
\end{abstract}

Keywords: Psychological tests; Professional qualification; Psychometrics.

\title{
Resumen
}

El objetivo principal de este estudio fue reflexionar sobre la aplicación de las pruebas psicológicas em uso em Brasil con las posibilidades de su validación por la Sociedad Brasileira de Psicología, destacando su utilidad y las principales incursiones em relación a su uso, especialmente em lo que se refiere a situaciones que evidencian trastornos relacionados con las dificultades de aprendizaje escolar em niños con inteligencia normal, a través de una evaluación integral que integra el funcionamiento intelectual y la organización de la personalidad. El fundamento teórico psicoanalítico sostiene que los procesos psíquicos patológicos pueden disminuir la función de la curiosidad y causar síntomas de inhibición intelectual o falta de concentración. El uso de instrumentos de evaluación psicológica es una práctica necesaria para el trabajo del psicólogo. Desde esta perspectiva, este estudio tuvo como objetivo investigar el uso de instrumentos de evaluación psicológica por parte de los psicólogos. En cuanto a los instrumentos más utilizados, se encontró que son pruebas de evaluación de la personalidad.

Palabras clave: Pruebas psicológicas; Formación profesional; Psicometría.

\section{Introdução}

Use o Muitos são os estudos relacionados as dificuldades de aprendizagem e os distúrbios que a rodeiam. O assunto em voga está relacionado a uma situação onde se percebe um déficit e que não é gerado momentaneamente, mas sim pela pouca capacidade de se ater a uma única situação que requer atenção do sujeito. Ainda há de se levar em conta que os problemas gerados a partir dessa situação necessariamente não necessitam ser tratadas com medicamentos ou com constantes acompanhamentos médicos, pois como as demais situações relacionadas a aprendizagem necessitam de ações dos professores e de sua equipe pedagógica para realizar atividades que garantam a melhoria da atenção dessas crianças.

Muitas são as causas que podem ser identificadas como geradoras dos problemas de aprendizagem e dentre eles estão classificados alguns distúrbios e um dos principais deles e mais comum nos dias de hoje é o Déficit de Atenção e Hiperatividade (TDAH) o qual tem como características básicas a desatenção, a agitação e a impulsividade.

Esse distúrbio tem se tornado constante e vem sendo um grande desafio para a educação, pois as crianças desse novo milênio apresentam uma personalidade muito mais agitada que anteriores, principalmente dado a fatores sociais e culturais a expansão dos meios de comunicação, até pelo pelas vantagens que a globalização e o processo de modernidade do vem oferecendo. Dessa forma surge a necessidade de se buscar mais informações sobre os reais problemas que levam aos distúrbios de aprendizagem evidenciando se possível suas causas e criando um planejamento eficaz para melhor conduzir o processo.

Os testes psicológicos têm papel relevante nesse processo, pois a partir deles é que se pode chegar a respostas que o professor em sala de aula não consegue ter. Nesse sentido é que existem diversos instrumentos que ajudam a diferenciar a 
situação que leva aos problemas relacionados com a leitura, escrita, concentração, apatia, hiperatividade e tantas outras situações comuns na escola.

Diante dessa necessidade, o estudo aqui apresentado esclarece sobre as características, consequências e validação dos diagnósticos, bem como suas implicações no ambiente escolar, apontando alternativas de intervenção em sala de aula dando-se sugestões de como atuar.

\section{Metodologia}

A presente pesquisa molda-se segundo a abordagem qualitativa, que para Bodgan e Biklen (1994) a realidade é enraizada nas percepções dos sujeitos, cujo intuito é interpretar e compreender significados através de narrativas e de observações no lugar de quantificações.

A investigação qualitativa normalmente ocorre em situações naturais e apresenta múltiplas características, podendo citar: usa múltiplos métodos de recolha de dados; há participação ativa do pesquisador; as questões a serem investigadas podem ser modificadas e redefinidas durante o desenvolvimento da pesquisa; é profundamente interpretativa e descritiva; sendo também indutiva; o investigador analisa os dados indutivamente; não havendo a preocupação em arranjar dados ou evidência para provar. Dessa forma, o investigador que utiliza a abordagem qualitativa preocupa-se mais com o processo e o contexto do que simplesmente com os resultados em si (Bogan \& Biklen, 1994).

A investigação também apresenta características descritiva e exploratória. A modalidade dessa investigação foi realizada pelas pesquisadoras por meio de um processo de observação não participante. A definição do problema é parte decisiva do planejamento de uma pesquisa, pois nos obriga a uma profunda reflexão. Do empenho na formulação do problema resulta o bom planejamento que facilitará a elaboração do trabalho. A metodologia descritiva observa, registra, analisa e correlaciona fatos ou fenômenos (variáveis) sem manipulá-los e procura ainda, descobrir, com precisão possível, a frequência com que um fenômeno ocorre, sua relação e conexão com outros.

\section{Resultados e Discussão}

Os profissionais da área de educação comumente enfrentam situações onde precisam lidar com alunos que apresentam os mais diversos históricos. Alguns possuem uma condição socioeconômica desfavorável, outros não recebem o incentivo correto para o estudo em casa e há ainda os que apresentam problemas de fundo biológico. Em relação e este último caso, os estudos se fundamentam, principalmente, no campo das dificuldades de aprendizagem.

Embora nem sempre tenha se declarado os distúrbios de aprendizagem sempre foram um grande nó no processo de ensino e aprendizagem, e muitos professores já passaram por sérios problemas para poder contorná-los. Basicamente trata-se de um campo tão complexo quanto fascinante, no qual a aprendizagem da leitura e da escrita tem sido objeto de vários estudos no mundo todo com conteúdo específicos.

Muitas são as variáveis de distúrbios apresentadas como causas dos problemas de déficit de aprendizagem, algumas delas dizem respeito às situações emocionais, porém em outros casos há probabilidade de genética alterada ou de distúrbios inerentes a situações cotidianas, sendo a dislexia um dos problemas mais comuns, contudo, nem sempre é levado em consideração pelos educandos e pais.

No ambiente escolar percebe-se que alguns profissionais atribuem a falta de atenção e a hiperatividade constante na vida das crianças e dos adolescentes como uma doença, a qual a pessoa adquire e não tem cura. Na verdade este é um processo genético, causado principalmente por fatores biológicos que levam ao problema citado. Porém, na nomenclatura e na prática os 
problemas gerados pela falta de atenção e hiperatividade são tratados como distúrbios, ou seja, uma ação desencadeada de outras, que acionam o mecanismo do "sempre alerta".

Evidentemente essas situações podem ser contornadas ou tratadas, contudo, a melhor prescrição que se faz, é a que estabelece um tratamento em forma de direcionamento das atividades propostas para estas crianças. Nesse sentido, na maior parte dos casos o tratamento requer atenção e assistência de profissionais da área de psicologia que desenvolvam os testes psicológicos e que a partir destes possam realizar o parecer concreto dos sintomas apresentados, bem como sugerir meios de contornar a situação, melhorando-as.

A fundamentação teórica psicanalítica sustenta que processos psíquicos patológicos podem diminuir a função da curiosidade e causar sintomas de inibição intelectual. Tarnopolsky (1995) expôs um histórico sobre o tema, ressaltando que inicialmente o fracasso escolar era explicado por uma associação direta a um déficit intelectual, ou seja, que os fracassos na aprendizagem eram devido a um baixo Q.I. e que, portanto, para uma adequada aprendizagem escolar bastava apenas um nível normal de inteligência.

Segundo a mesma autora, esta visão da esta visão da problemática alterou-se principalmente a partir das contribuições de abordagem psicanalítica, entre elas, os trabalhos de Melanie Klein (1981) sobre os efeitos da inibição da curiosidade no desenvolvimento intelectual da criança. O trabalho de Klein foi desenvolvido a partir do teste CAT-A que mede os elementos inibidores em relação as atividades a serem desenvolvidas. o Teste de Apercepção Temática Infantil (CAT-A), em dois trabalhos anteriores, para investigar a psicodinâmica e a influência dos distúrbios emocionais nesse quadro clínico (Paulo, 1998; Tardivo \& Paulo, 1992). Os resultados obtidos evidenciaram crianças com dificuldade em tolerar frustrações, fragilidade das funções egóicas, organização defensiva imatura, impossibilidade de solucionar conflitos, tendência à introversão, sentimentos de insatisfação, insegurança e inadequação. Os psicodinamismos emocionais ligados à dificuldade em lidar com a agressividade geram ansiedade e tensão internas, levando, por sua vez, a uma ineficiência temporária das funções mentais desenvolvidas e empobrecendo a produção dessas crianças.

Em trabalho recente, Benczik (2005) utilizou o CAT-A para investigar os aspectos psicodinâmicos envolvidos no Transtorno de Déficit de Atenção/Hiperatividade (TDAH) em meninos com inteligência normal. Também usou a teoria das Relações Objetais, de Klein, como suporte de entendimento. A autora afirmou que o CAT-A foi eficiente para diferenciar as crianças com e sem TDAH. Os resultados principais da análise feita às cegas identificou entre os meninos com o transtorno: negação maníaca onipotente, trapaça, sentimentos derivados do instinto.

Outro estudo também realizado nesse mesmo sentido é com a aplicação do teste WISC III onde a abordagem tem fundamentação teórica psicanalítica das relações objetais que sustenta a interpretação de que processos psíquicos patológicos podem diminuir a função da curiosidade e causar sintomas de inibição intelectual.

O WISC III é Instrumento clínico muito utilizado a nível internacional para avaliação individual da capacidade intelectual de crianças e adolescentes (de 6 a 16 anos). Possui vários sub testes, que correspondem a diferentes aspectos da inteligência, determinando três medidas compostas: QIs Verbal, de Execução e Total que oferecem estimativas das capacidades intelectuais dos indivíduos. Tanto o CAT-A como o WISC III, são bem aceitos na área e tem correspondido as expectativas dos profissionais, porém, para melhores resultados ambos os testes foram adaptados para a clientela brasileira.

Ainda pode-se citar uma determinada quantidade de outros testes que que tem o propósito de mensurar a capacidade intelectual do indivíduo e os fatores inibidores do conhecimento.

Testes estes que buscam entender as principais situações que levam ao déficit de atenção a aos problemas de aprendizagem. (Padilha, 2207 p.76), reitera que: 
Tendo em vista o interesse na identificação dos testes psicológicos mais utilizados, observou-se que entre os dez testes mais utilizados pelos participantes, seis deles avaliam características de personalidade (HTP - House, Instrumentos de Avaliação Psicológica: Uso e Parecer, Tree, Person, IFP - Inventário Fatorial de Personalidade, TAT - Teste de Apercepção Temática, Teste de Wartegg, MACHOVER - Teste da Figura Humana, e o Teste Gestaltico Viso-Motor Bender); os demais são instrumentos de avaliação da inteligência e/ou aptidões (Matrizes Progressivas de Raven, Desenho da Figura Humana, AC Atenção Concentrada e a Escala de Transtorno do Déficit de Atenção/ Hiperatividade).

Ao mesmo tempo em que não são decisivos para o diagnóstico, os testes psicológicos acabam sendo de grande ajuda para o profissional. Todavia, ainda há uma grande luta no sentido de desburocratizar a sua utilização, pois muitos apresentam somente a versão americana o que não corresponde as mensurações utilizadas no Brasil.

A dificuldade em serem instrumentos confiáveis para diagnóstico reside no fato de que muitas crianças obtêm resultados que não revelam suas dificuldades, pelo fato de o teste ser vivenciado como novidade ou por se beneficiarem da relação um a um com o aplicador (Hallowell \& Ratey, 1999).

Um outro fator muito importante que dificulta atualmente o uso de testes psicológicos no processo diagnóstico é que muitos deles ainda não estão aprovados pelo Conselho Federal de Psicologia (CFP), o que restringe seu uso quase que exclusivamente para a pesquisa.

Noronha (2002), relata que dentre os testes que os psicólogos de São Paulo, mais fazem uso, se evidenciam os que objetivem avaliar a personalidade, sendo que os que tratam de mensurar a inteligência são pouco usados. Entretanto, em relação a aprendizagem, um dos testes mais utilizados está relacionado ao desempenho em escrita, através de um ditado padronizado (ADAPE) elaborado e padronizado por Sisto (2002B) e que evidencia as dificuldades mais comuns na escrita de crianças.

\section{Considerações Finais}

Os testes psicológicos comumente usados têm como princípio buscar respostas para as situações de ansiedade e de inquietação de pais e professores em relação as crianças e seu desenvolvimento escolar. Muitas são as ações que levam a pesquisas, crianças retraídas, apáticas, eufóricas demais, que apresentam dificuldade na aprendizagem e as que denotam um elevado conhecimento para a série que cursam ou para a sua idade, ou ainda as situações relacionadas com casos de crianças que denotavam certa esperteza e agilidade maior que a maioria e as quais eram chamadas de "bicho carpinteiro", todas estas situações precisam ser conhecidas e a partir do trabalho do psicólogo espera-se encontrar os familiares e a escola espera encontrar respostas para que possam realizar as devidas intervenções.

Desse modo é que tais profissionais lançam mão de testes avaliativos que tem como objetivo, conhecer a real situação do aluno.

Porém, percebe-se que estas ações são por vezes um problema para o psicólogo, pois nem todos os testes mais comuns e que estão licenciados para o uso pelo Conselho de Psicologia Brasileiro correspondem as necessidades a serem aferidas, outros ainda, apresentam um programa fora da realidade da maioria da clientela brasileira, sendo que o padrão de mensuração não condiz com a realidade dos avaliados.

Em outras situações os testes precisam ser adaptados para uma clientela mais velha, pois em tempos atuais já é comum perceber os sintomas que necessitam de acompanhamento também em indivíduos tidos como normais, porém o que classifica a hiperatividade é o grau de intensidade que se fazem presente na rotina dos sujeitos, sendo comum aos hiperativos que o excesso de distração apresente prejuízos que acarretam problemas ou embaraços ao longo da vida, sendo mais difícil de adequar os testes existentes. 
Dessa maneira, mesmo que os testes de avaliações apresentem uma boa contribuição de valor científico, vale reiterar que a ação do psicólogo é de suma importância, principalmente em relação a percepção dos sentimentos e interpretação da subjetividade para melhor entendimento do desempenho acadêmico da criança para aprofundar a compreensão dos mecanismos envolvidos nos quadros de inibição intelectual.

Cabe aqui colocar que este trabalho pode vir a contribuir com futuras pesquisas sobre a temática em questão. Por fim, sugerimos para trabalhos futuros que tragam em pauta demais transtornos de aprendizagem que as pessoas sofrem atualmente, para além dos que foram trazidas neste estudo. Sendo importante também refletir a respeito do não diagnóstico, pois o sujeito que possui um transtorno de aprendizagem não diagnosticado acaba sofrendo não só em razão de sua dificuldade. Ainda é interessante discutir sobre as diferenças entre TDAH, dislexia, déficit de atenção e hiperatividade já que muitos indivíduos facilmente as confundem devido aos sintomas serem bastante semelhantes.

\section{Referências}

Abrahão, N. da S. \& Fantacini, R. A. F. (2017). Transtorno do Déficit de Atenção com Hiperatividade (TDAH): desafios e possibilidades frente a sala de aula. Research, Society and Development, 6(3), 1-13.

Benczik, E. B. P. (2005). Crianças com Transtorno de Déficit de Atenção/Hiperatividade: um estudo dos aspectos psicodinâmicos a partir de Teste de Apercepção Infantil - CAT-A. (Tese de Doutoramento). Instituto de Psicologia, Universidade de São Paulo, São Paulo.

Bogdan, R. \& Biklen, S. (1994). Investigação qualitativa em educação. Porto Editora.

Carlotto, M. S., \& Câmara, S. G. (2020). Escala de Avaliação da Síndrome de Burnout em Estudantes Universitários: construção e evidências de validade. Research, Society and Development, 9(7), 1-23.

Carvalho, A. dos S. M. de., Pereira, P.C., Oliveira, M. M. de. (2021). Déficit de Atenção, Hiperatividade, Desordem, Legislação, Escola e PAE - Breve análise. Research, Society and Development, 10(14), 1-8.

Cunha, C. A. da, Sisto, F. F. \& Machado, F. (2006) Dificuldade de Aprendizagem na Escrita e o Autoconceito num Grupo de Crianças. Avaliação Psicológica, $5(2), 153-157$.

Hasbun, A. S. P. DE S., Formiga, N. S. \& Estevam, I. D. (2021). Teste z e tomada de decisão: avaliação psicológica ideográfica para manuseio de armas de fogo. Research, Society and Development, 10(4), 1-17.

Hallowell, E. M. \& Ratey, J. (1999). Tendência à distração: identificação e gerência do distúrbio do déficit de atenção da infância à vida adulta. Rio de Janeiro: Rocco.

Maffini, G., \& Cassel, P. A. (2020). O processo de avaliação psicológica: estudo de caso. Research, Society and Development, 9(5), 1-12.

Miranda, A. C. de, Gomes, G. M., Furtado, M. \& Figueiredo, V. L. (2004). Perfil intelectual de crianças com dificuldades de aprendizagem, avaliado pelo Teste WISC-III: um estudo exploratório. In: Congresso Nacional da sociedade brasileira de Rorschach e Métodos Projetivos. Anais. Porto Alegre: Sociedade Brasileira de Rorscharch, 3, 132.

Morais, M. R. R. \& Gontijo, R. A. G. (2021). A importância da entrevista na avaliação psicológica em contexto de trânsito. Research, Society and Development, 10(13), 1-6.

Noronha, A. P. P. \& Alchieri, J. C. (2004). Conhecimento em Avaliação Psicológica. Estudos de Psicologia, 21(1), 43-52.

Noronha, A. P. P. (2002). Os problemas mais graves e mais frequentes no uso dos testes psicológicos. Psicologia Reflexão e Crítica, 15(1), 135-142.

Padilha, S. (2007). Instrumentos de avaliação psicológica: uso e parecer de psicólogos. Avaliação Psicológica, 2007, 6(1), 69-76.

Paulo, M. S. L. de. (2008). Análise clínica da avaliação cognitiva de crianças com inibição intelectual. Psicólogo informação, 12 (12), 53-71.

Pedroso, L. V., Graup, S., Balk, R. de S., Castro, C. J. de, \& Arend, M. H. R. de F. (2021). The influence of Attention Deficit Hyperactivity Disorder (ADHD) on children's learning: An Integrative Literature Review. Research, Society and Development, 10(7), 1-12.

Tardivo, L. S. de L. P. C. (1992). Teste de Apercepção Infantil com Figuras de Animais (CAT-A) e Teste das Fábulas de Duss: estudos normativos e aplicações no contexto das técnicas projetivas. (Tese de Doutoramento). Instituto de Psicologia, Universidade de São Paulo, São Paulo.

Tarnopolsky, B. E. E. (1995). As técnicas projetivas no diagnóstico das dificuldades de aprendizagem. In: OCAMPO, M. L. S. et al. (1995). O processo psicodiagnóstico e as técnicas projetivas. 8. ed. São Paulo: Martins Fontes.

Santana, A. C., Pires, G. C. \& Leite, U. do R. (2021). Escala de construção da violência psicológica contra universitários. Research, Society and Development, $9(11), 1-25$.

Silva, M. de O., Santos, A, D. dos., Souza, V. R. N. de., Silva, E. D. S. da., \& Damasceno, R. O. (2021). Avaliação e atendimento psicológico na pandemia Covid-19 no Brasil: uma sistemática. Research, Society and Development. 10(12), 1-13.

Silva, W. S. da., Dourado, J. L. G. \& Ribeiro, J. G. B. (2021). Investigações no campo da Neuropsicologia: aspectos peculiares do transtorno do déficit de atenção com hiperatividade. Research, Society and Development, 10(11), 1-8. 